\title{
Application of Kinect Technology in Blind Aerobics Learning
}

\author{
https://doi.org/10.3991/ijet.v12.i12.7960 \\ Hui Qu \\ Hunan Institute of Engineering, Hunan, China \\ huiqu@hnie.edu.cn
}

\begin{abstract}
In order for blind people to learn aerobics more conveniently, we combined Kinect skeletal tracking technology with aerobics-assisted training to design a Kinect-based aerobics-assisted training system. Through the Kinect somatosensory camera, the feature extraction method and recognition algorithm of sign language are improved, and the sign language recognition system is realized. Sign language is translated through the sign language recognition system and expressed in understandable terms, providing a sound way of learning. The experimental results show that the system can automatically collect and recognize the aerobics movements. By comparing with the standard movements in the database, the system evaluates the posture of trainers from the perspectives of joint coordinates and joint angles, followed by the provision of movements contrast graphics and corresponding advice. Therefore, the system can effectively help the blind to learn aerobics.
\end{abstract}

Keywords-Kinect, Human-Computer Interaction, Posture Recognition, Occlusion Information Restoration, Blind Aerobics

\section{Introduction}

In the current process of aerobics training, learners reach the goal of exercise usually by repeatedly watching the video or by consulting coaches [1]. This is not only time- and effort- consuming, but brings great challenges to the blind. The meaning of our research is to combine aerobics training with Kinect skeletal tracking technology, such that the postures and movements can be captured and matched up with standard movements. Subsequently, the differences in the skeletal direction are analyzed and showed to blind people in a straightforward way, enabling them to find the gaps [2]. This method allows blind aerobics coaches and learners with high demand for teaching/self-teaching to make accurate adjustments of movements and postures that used to be below standard, so as to meet the standard of correctness. Moreover, the training process can begin at any time and in any place, which broadens teaching means. Trainers can foster their rhythms through the interaction with the computer. With our approaches, the digitalization and informatization of basic aerobics training and other training levels can be further developed [3-5]. 


\section{$2 \quad$ Literature review}

Human-computer interaction is defined as a technology that realizes the dialogue between human and computer in effective ways and that studies computer, human kinds, and their mutual influence. This technology is closely related to the research fields like artificial intelligence, machine vision, ergonomics, and cognitive psychology. Currently, more than 60,000 researchers worldwide are engaged in humancomputer interaction technology, and on annual average, there are more than 50 conferences related to this technology and over 400 articles published in magazines. The number of related research results exhibited in conferences has exceeded 3,000.

Foreign researchers have also been committed to human-computer interaction in multiple channels and other comprehensive ways. The Coyote robot developed by Dennis et al. [6], a professor of mechanical engineering at Virginia Tech, has been able to combine various modes of interaction information and take voice and hand gesture actions as the main interaction mode to achieve satisfactory performance results. Researchers such as Fritsch [7] have realized the interaction object tracking based on face and foot recognition. Stiefelhagen et al. [8], professor at the Karlsruhe Institute of Technology in Germany, has demonstrated the robustness of a multi-way interactive system through several experiments. The specific test equipment is to integrate hand gesture and voice into the ARMAR II and III robotic platform to establish a natural human-computer interaction system. Researchers at the University of Dublin, including Han [9], designed speech recognition, sight recognition, and face recognition in the Lego robot platform in order to enhance the naturalness of humancomputer interaction.

With the development of information technology and computer technology, multimodal human-computer interaction technology has been gradually developed to incorporate more than two kinds of recognition technologies. At present, most of the related research materials for multi-modal human-computer interaction involve sound recognition technology and limb movement recognition technology $[10,11]$, and their common features are intuitive, interface-friendly and information-rich. Therefore, the body movement interaction technology is closer to the development direction of interactive technology, arising as the core technology of multi-mode human-computer interaction. Its development is directly related to the future of human-computer interaction.

\section{Methods}

\subsection{Kinect-based human joint recognition}

Kinect recognizes and tracks human skeleton. It identifies the coordinates of the 25 joints of the human body, establishes the human skeleton structure, and combines the depth information to represent the human skeleton structure in three-dimensional space. The human joint recognition includes the following three parts: (1) remove the background; first, find the possible position of human body; then, use the edge detec- 
tion method to extract the outline of the human body according to the distance detected by the Kinect distance sensor. (2) Identify the important parts of the human body, including the head, arms, legs, and trunk. (3) Identify human joints; connect the joints in Kinect and analyze the front and side joints to judge where the human body is, as shown in Figure 1.

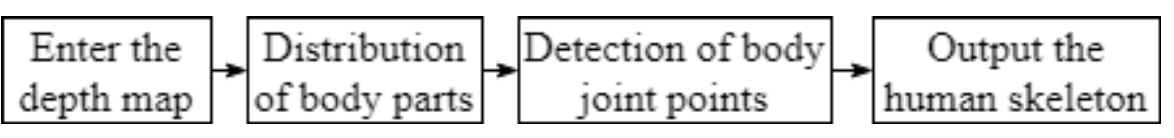

Fig. 1. Steps of human skeleton joints recognition

\subsection{Human skeletal length ratio}

A moving human body is vulnerable to the external environment, softness of clothes, and other factors. In many cases, the contour information does not well reflect the details of human motion. The skeleton can not only represent the topology of human body, but also retains the body's geometric information. As a result, a large number of skeleton-based human recognition techniques have emerged, including bone extraction, target matching, and motion capture [55]. In this paper, the human body is expressed as a whole which is rigidly connected by multiple joints. Each joint is connected by a rigid body. Each joint represents a characteristic point, and the length of the rigid connection is unchanged. Therefore, Human movement is simplified as the movement of the human skeleton. The main male skeletal length is shown in Table 1.

Table 1. Standard male skeletal length

\begin{tabular}{|l|c|}
\hline \multicolumn{1}{|c|}{ Skeleton } & Length (cm) \\
\hline Right upper arm & 27 \\
\hline Right lower arm & 33 \\
\hline Left upper arm & 27 \\
\hline Left lower arm & 33 \\
\hline Right thigh & 45 \\
\hline Right calf & 44 \\
\hline Left thigh & 45 \\
\hline Left calf & 44 \\
\hline
\end{tabular}

\subsection{Judgement of the occluded joint point}

During the movement of the human body, it often happens that the joint point is blocked by other parts of the body. Therefore, Kinect cannot capture all the joint points or will wrongly recognize the non-joint points. In this paper, the occluded points are repaired according to the continuity of human motion and the degree of freedom of the joints. According to the results of several experiments, Kinect can accurately obtain the information of the trunk points of the human body, but the trunk 
is easily separated from the limbs. For example, the wrist and the ankle are disconnected from the body trunk. This article assumes that Kinect collect credible information of head, neck, shoulder and other joints. Accordingly, the only work to do is to verify the information about wrist, elbow, knee and ankle. The flow chart to judge the occluded skeleton points are shown in Figure 2 below.

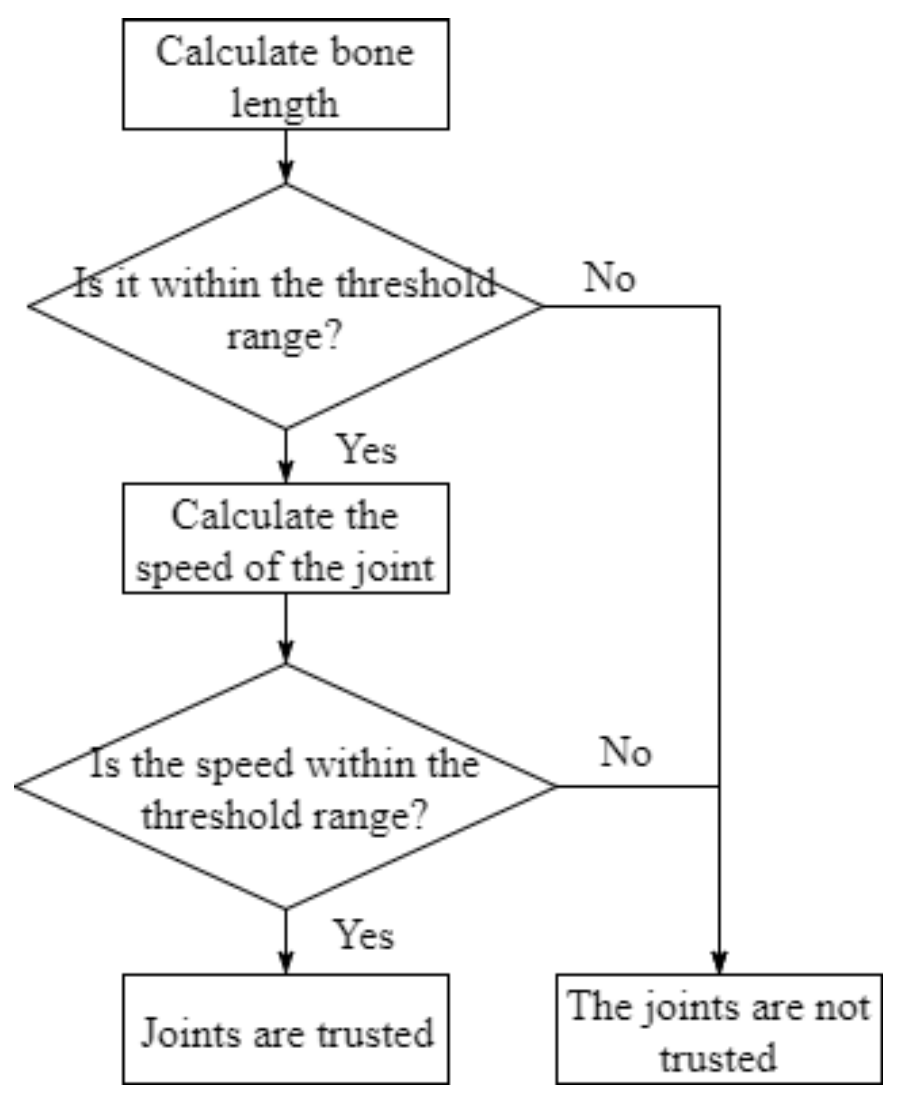

Fig. 2. Flowchart of judging the credibility of occluded skeleton joints

\subsection{Kinect-based aerobics auxiliary training system}

Data acquisition, data processing and data analysis are the main part of the system. The collected data includes the aerobics coach's and trainers' movement data. Through the acquisition of aerobics coach movement information, we establish a standard aerobics posture database. Data processing refers to the restoration of occluded joint points to obtain the complete human skeleton information. The data analysis is to compare the trainers' movement information with the standard movement information and provide the training guide according to the comparison result so as to quickly improve the trainers' exercise effect. The corresponding system framework is shown in Figure 3. 


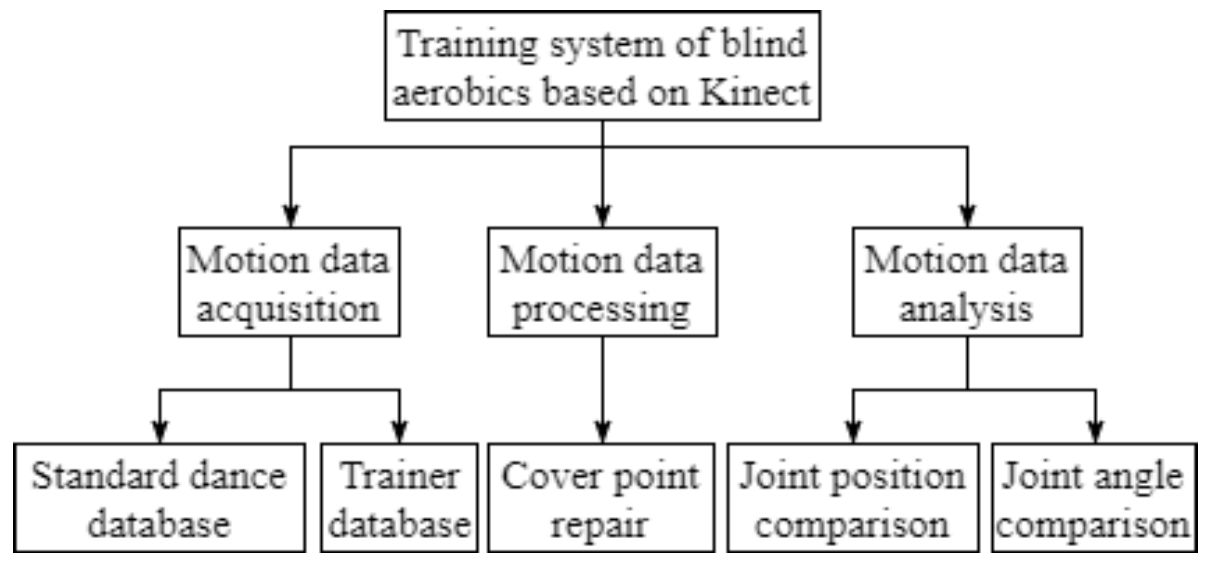

Fig. 3. . Block diagram of dancing auxiliary training system based on Kinect

Before model training, it is necessary to set the values for each element in the model. Due to the small number of training samples, the model may possibly be less accurate if with too many states. In this system, ten dynamic hand sign language of "material", "super", "adjustment", "struggle", "competition", "hold", "magnificent", "publication", "friend" and "teaching" are recognized, and the state values are tested out when each of them remains at the optimal recognition rate. The relationship between state and recognition results is shown in Figure 4. Table 2 shows the best state of each sign language.

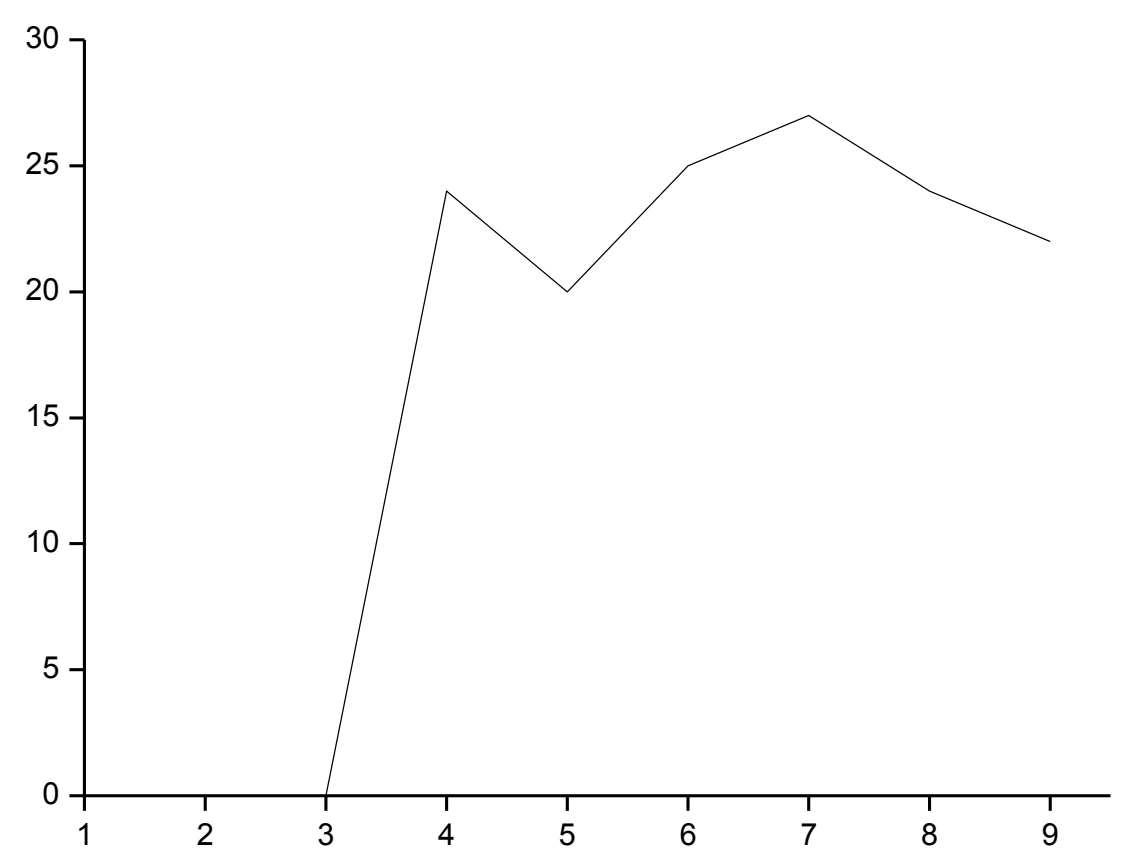

Fig. 4. Relation between state and recognition 
Table 2. The best states of sign language

\begin{tabular}{|l|l|l|l|l|l|}
\hline Sign language & Material & Super & Adjustment & Struggle & Game \\
\hline Status value & 4 & 5 & 9 & 8 & 4 \\
\hline Sign language & Held & Magnificent & Publish & Friend & Teaching \\
\hline Status value & 7 & 7 & 8 & 4 & 3 \\
\hline
\end{tabular}

\subsection{Sign Language Recognition System Design and Implementation}

The sign language recognition system promotes the communication between blind people and able-bodied people by reducing communication barriers. When applied in schools, the sign language recognition system enables the blind to communicate better and more with the normal and live and study healthier. Meanwhile, it provides a new access to the implementation of man-computer interaction theories. The flow chart of the sign language recognition system is shown in Figure 5.

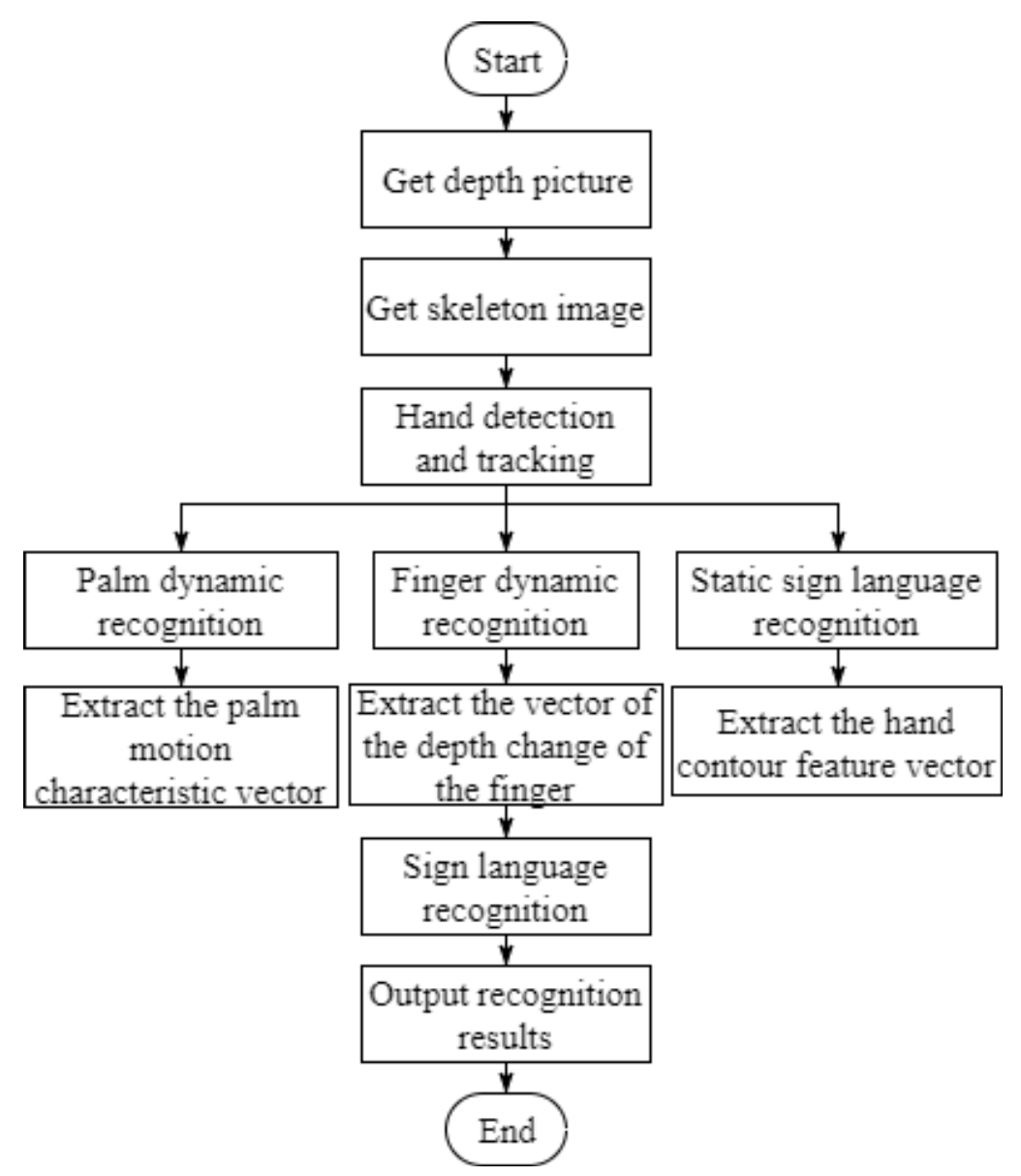

Fig. 5. The flow chart of sign language recognition system 
This study mainly uses Kinect somatosensory equipment to recognize human sign language. The hand tracking and identification process is shown in Figure 6.

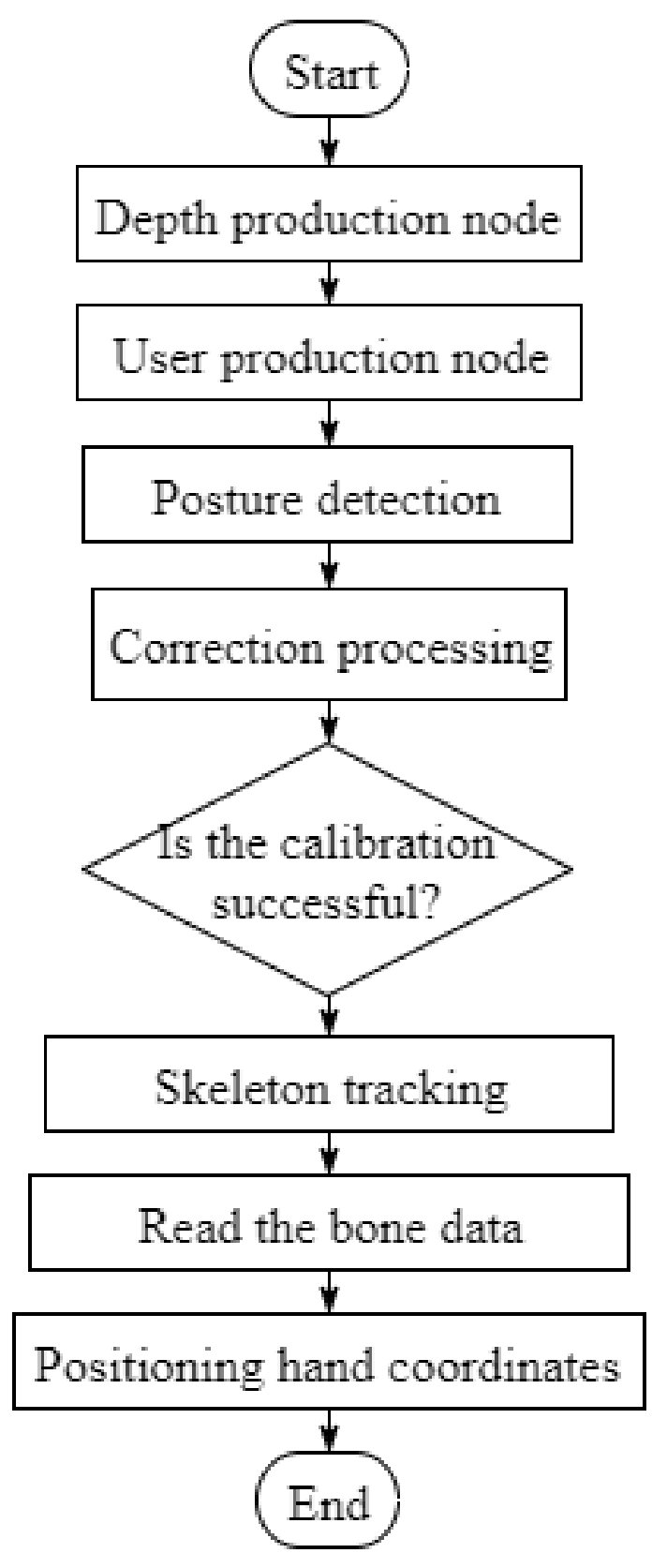

Fig. 6. Hand tracking and identification process 


\section{Conclusion}

In this paper, the human detection and tracking technology is researched, and the fixed-axis-based joint angle representation method is proposed. It improves the way to recognize postures in joint terms, identifying human movement accurately. The blind aerobics auxiliary training system is designed, which adds visible prompts of wrong correction. The authors also introduce the methods to extract feature vectors in three scenarios: palm movement, finger movement and static sign language. Finally, on the basis of the aforementioned research, the Kinect-based sign language recognition system is designed, developed and implemented. The experiment results show that this system can show the accurate difference between the trainers' and the standard movements, enabling trainers to adjust movements and postures so as to raise the level of aerobics. In this way, our system reaches the goal of auxiliary training.

\section{$5 \quad$ References}

[1] Huang, H. C., Wong, M. K., Yang, Y. H., Chiu, H. Y., \& Teng, C. I. (2017). Impact of playing exergames on mood states: a randomized controlled trial. Cyberpsychology, Behavior, and Social Networking, 20(4), 246-250. https://doi.org/10.1089/cyber.2016.0322

[2] Hasselmann, V., Oesch, P., Fernandez-Luque, L., \& Bachmann, S. (2015). Are exergames promoting mobility an attractive alternative to conventional self-regulated exercises for elderly people in a rehabilitation setting? Study protocol of a randomized controlled trial. BMC geriatrics, 15(1), 108. https://doi.org/10.1186/s12877-015-0106-0

[3] Kayama, H., Okamoto, K., Nishiguchi, S., Yukutake, T., Tanigawa, T., Nagai, K., \& Aoyama, T. (2013). Efficacy of an exercise game based on Kinect in improving physical performances of fall risk factors in community-dwelling older adults. GAMES FOR HEALTH: Research, Development, and Clinical Applications, 2(4), 247-252. https://doi.org/10.1089/g4h.2013.0006

[4] Kimhy, D., Vakhrusheva, J., Bartels, M. N., Armstrong, H. F., Ballon, J. S., Khan, S., \& Castrén, E. (2015). The impact of aerobic exercise on brain-derived neurotrophic factor and neurocognition in individuals with schizophrenia: a single-blind, randomized clinical trial. Schizophrenia bulletin, 41(4), 859-868. https://doi.org/10.1093/schbul/sbv022

[5] Kimhy, D., Vakhrusheva, J., Bartels, M. N., Ballon, J. S., Castrén, E., \& Sloan, R. P. (2017). 398. Aerobic Exercise Training in People with Schizophrenia: Neural, Cognitive, and Functional Benefits. Biological Psychiatry, 81(10), S162-S163. https://doi.org/10.1016/j.biopsych.2017.02.415

[6] Lohse, K. R., Hilderman, C. G., Cheung, K. L., Tatla, S., \& Van der Loos, H. M. (2014). Virtual reality therapy for adults post-stroke: a systematic review and meta-analysis exploring virtual environments and commercial games in therapy. PloS one, 9(3), e93318. https://doi.org/10.1371/journal.pone.0093318

[7] Lazzari, R. D., Politti, F., Belina, S. F., Collange Grecco, L. A., Santos, C. A., Dumont, A. J. L., ... \& Santos Oliveira, C. (2017). Effect of transcranial direct current stimulation combined with virtual reality training on balance in children with cerebral palsy: a randomized, controlled, double-blind, clinical trial. Journal of motor behavior, 49(3), 329336. https://doi.org/10.1080/00222895.2016.1204266 
[8] Mereuta, C., Ganea, D., \& Mereuta, E. (2014). The influence of spatial perception on control and energetic parameters. Procedia-Social and Behavioral Sciences, 137, 50-56. https://doi.org/10.1016/j.sbspro.2014.05.251

[9] Singh, A., Piana, S., Pollarolo, D., Volpe, G., Varni, G., Tajadura-Jiménez, A., \& BianchiBerthouze, N. (2016). Go-with-the-flow: tracking, analysis and sonification of movement and breathing to build confidence in activity despite chronic pain. Human-Computer Interaction,31(3-4),335-383. https://doi.org/10.1080/07370024.2015.1085310

[10] Sheehy, L., Taillon-Hobson, A., Sveistrup, H., Bilodeau, M., Fergusson, D., Levac, D., \& Finestone, H. (2016). Does the addition of virtual reality training to a standard program of inpatient rehabilitation improve sitting balance ability and function after stroke? Protocol for a single-blind randomized controlled trial. BMC neurology, 16(1), 42. https://doi.org/10.1186/s12883-016-0563-x

[11] Guo, K. (2016). Empirical Study on Factors of Student Satisfaction in Higher Education. Revista Iberica de Sistemas e Tecnologias de Informacao, E11, 344-355.

\section{Author}

Hui Qu is with Hunan Institute of Engineering, Hunan, China.

Article submitted 05 November 2017. Published as resubmitted by the uthors 16 December 2017. 